International Journal of Cardiology and Research (IJCRR)

ISSN 2470-4563

\title{
"Role of D-Dimer in One Year Recurrence of Venous Thromboembolism after Completed Anticoagulation Treatment" Following A First Idiopathic Deep Vein Thrombosis
}

Research Article

\author{
Tamizifar $\mathrm{B}^{1}$, Padideh $\mathrm{O}^{1}$, Esfahani $\mathrm{MA}^{2 *}$
}

${ }^{1}$ Department of Internal Medicine, St.Alzahra Hospital, Isfahan University of Medical sciences, Isfahan, Iran

${ }^{2}$ Department of cardiology, St.Alzahra Hospital, Isfahan University of Medical sciences, Isfahan, Iran

\begin{abstract}
After oral anticoagulant therapy (OAT) withdrawal, the recurrence of risk is greatest in the first year and gradually diminishes, while the increased bleeding risk may offset the benefits of prolonged OAT. the benefits of extending anticoagulation should be balanced against the risk of bleeding . $\mathrm{D}$-dimer assay and measurement of residual thrombosis on ultrasonography, so-called residual vein obstruction (RVO) were proposed to be effective in selecting patients with idiopathic DVT

Materials and methods: This single center prospective study was conducted in patients with a first episode of symptomatic proximal DVT, detected by compression ultrasonography (CUS), and who received OAT for at least 3 months. Follow-up was extended from Oct. 2011 , to Aug. 2013. After enrollment, patients were re-evaluated at $1 \mathrm{st}$, 6th and 12th months of their follow-up. At the first and 30-day visits, venous blood samples were taken for D-dimer test. All enrolled patients without RVT were stopped OAT.

At each follow-up visit, we examined patients and inquired about health status, clinical symptoms or signs of recurrent VTE, bleeding, post-thrombotic manifestations, adherence to treatment, and concomitant analgesic or anti-inflammatory therapy.

The end point outcomes were VTE recurrence or complete of this survey follow-ups.

Results: A total of 68 eligible patients were enrolled. Four patients (two patients need to using long-term oral anticoagulation, and another two patients for lost to their first follow-up) were excluded. D-dimer and CUS at first was normal in 28 patients (44\%) while the remaining 36 patients had abnormal D-dimer but normal CUS while both groups were not consuming their anticoagulant.

Total follow-up was 52.5 patient-years. Median follow-up was 11 months (95\% CI: 6-17 months). A minimum follow-up of 12 months was available in 44 patients (68\%). During follow-up, three recurrent events were recorded (4.6\% of patients - 95\% CI: 3-7\%). All Recurrent events were ipsilateral DVT. Among these index cases, all had an abnormal D-dimer at either T0 and/or T1. The recurrence rate was higher in males than in females ( $(8.6 \%$ vs. $2.2 \%)$ with an abnormal D-dimer at T0 and/or T1 with a multivariate hazard ratio of $2.1(95 \% 1.2-7$; $\mathrm{p}=0.04)$. Patients older than 65 years had a higher rate of events than younger patients (2/ 12: (16\%); 95\% CI: 4-24\% Vs. 1/ 52: (2\%); $95 \%$ CI: $0.05-3 \%)$ and hazard ratio was about 3.8; (95\% CI: 1.95-8.4 p= 0.04). Patients with recurrences had higher mean D-dimer at both T0 and T1 when compared with those without recurrences but the difference was significant only for D-dimer at T1. During follow-up, two patients died (3\%).

Conclusion: In our cohort of DVT patients, the annual risk of recurrence with an abnormal D-dimer, either during or at one month after VKA withdrawal, was $4.6 \%$ which is much lower to the annual risk of recurrence in most studies with idiopathic and provoked VTE. The benefits of extending anticoagulation should be balanced against the risk of bleeding. So according to these results of D-Dimer assay before withdrawal of OAT, this test is helpful for OAT withdrawal decision making.
\end{abstract}

Key Words: Deep Vein Thrombosis; Recurrent Venous Thromboembolism; Residual Vein Obstruction; D-Dimer.

\section{*Corresponding Author:}

Morteza Abdar Esfahani,

Cardiologist,Advanced Echocardiologist, Department of cardiology, St. Alzahra Hospital, Isfahan University of Medical sciences, Isfahan, Iran. Tel: +98-311-6685555; +989133107738, Fax: +98-311-6684510

E-mail: abdariranian@yahoo.com

Accepted: April 29, 2014

Published: April 30, 2014

Citation: Tamizifar B, Padideh O, Esfahani MA (2014) Role of DDimer in One Year Recurrence of Venous Thromboembolism after Completed Anticoagulation Treatment" Following A First Idiopathic Deep Vein Thrombosis. Int J Cardiol Res. 1(2), 4-8. doi: http://dx.doi. org/10.19070/2470-4563-140002

Copyright: (C) 2014 Morteza Abdar Esfahani. This is an open-access article distributed under the terms of the Creative Commons Attribution License, which permits unrestricted use, distribution and reproduction in any medium, provided the original author and source are credited.

\section{Introduction}

Approximately 900,000 people are diagnosed with a venous thromboembolism (VTE) annually with 5\% Americans experiencing a Deep vein thrombosis (DVT) during their lifetime. [1] Immediate mortality associated with DVT has been estimated at $5 \%$, but Cumulative mortality in the first 6 months after a thromboembolic event is $5-10 \%$ increasing to $20 \%$ by 2 years, mainly because of malignancy [2].

Under-anticoagulation with a Vitamin $\mathrm{K}$ antagonist (VKA), increases the risk of thrombotic events, and over-anticoagulation increases the risk of serious bleeding. The narrow therapeutic range of VKAs is further complicated by numerous drug, diet, and metabolic interactions [2]

In patients with unprovoked VTE, Long-term anticoagulant treatment is highly effective in preventing recurrent VTE, the risk of a 
recurrent event in the first year following discontinuation of anticoagulation varies from $5 \%$ to $15 \%$, and such duration of therapy is controversial but the optimal duration of this therapy remains uncertain. After oral anticoagulant therapy (OAT) withdrawal, the recurrence of risk is greatest in the first year and gradually diminishes, while the increased bleeding risk may offset the benefits of prolonged OAT $[3,4]$. After the first three months, the American College of Chest Physicians (ACCP) recommends an individual evaluation for the risk-benefit ratio of long-term therapy in patients with unprovoked VTE for their relevant risk of recurrence [5]. So, the benefits of extending anticoagulation should be balanced against the risk of bleeding.[6]

New parameters have, however, been proposed to optimize OAT duration; among them, D-dimer assay and measurement of residual thrombosis on ultrasonography, so-called residual vein obstruction (RVO) were proposed to be effective in selecting patients with idiopathic DVT who may benefit from a prolonged anticoagulation $[3,4]$

D-dimer levels were shown to have a high negative predictive value for recurrent VTE. So far only one randomized trial, the ROLONG study, has been performed to establish the role of Ddimer for assessing the risk of recurrence after a first episode of VTE.[6] On the other hand, RVO might impair venous flow, leading to stasis and activation of the coagulation cascade, or be a surrogate marker for a hypercoagulable state. Whereas some previously published studies have shown an association between RVO and recurrent VTE, others have not. It is unknown D-dimer could be a predictor of cardiovascular events and occult cancer in subjects with a first episode of idiopathic proximal deep vein thrombosis (DVT). [3], [6-8].

The aim of the present study was to assess the predictive value of D-dimer for recurrent VTE.

\section{Methods}

\section{Study patients}

Patients with a first episode of documented idiopathic proximal DVT were eligible for the study if they had completed at least 3 months of oral anticoagulant therapy (OAT) with reaching target INR (international normalized ratio) between 2.0 and 3.0.

Idiopathic DVT was defined as a thrombosis episode in apparently healthy individuals with the absence of pregnancy or puerperium, recent fracture or plaster casting of a leg, immobilization with confinement to bed for 3 consecutive days, active malignan- cy, known thrombophilic states, using precipitating drugs and surgery with general anesthesia lasting more than 30 minutes during the last three months.

Exclusion criteria were patients with secondarily DVT such as severe liver or renal insufficiency (glomerular filtration rate $<30$ ), antiphospholipid antibody syndrome, other known thrombophilic states (such as deficiencies of antithrombin and protein $\mathrm{C}$ and $\mathrm{S}$, homozygous for FV Leiden or prothrombin gene (20210G_A) mutations, coexistence definite indications for cardiac or cerebral for using anticoagulation, limited life expectancy, or those who lived too far from the research center.

The duration of anticoagulation based on compression ultrasonography and d-dimer study was approved by our university research committee. All enrolled patients provided written informed consent.

\section{Study design}

This single center prospective study was conducted in patients with a first episode of symptomatic proximal DVT, detected by compression ultrasonography (CUS), and who received OAT for at least 3 months. Those who agreed to participate in the study underwent an examination to assess baseline clinical conditions and to exclude contraindications. Study enrollment started on September 2011 and ended on $30^{\text {th }}$ June 2012. Follow-up was extended from $30^{\text {th }}$ October 2011, to $21^{\text {st }}$ August 2013. After enrollment, patients were re-evaluated at $1^{\text {st }}, 6^{\text {th }}$ and $12^{\text {th }}$ months of their follow-up. (Fig. 1)

On the day of VKA discontinuation, CUS of the lower limbs was performed by investigators unaware of the laboratory findings of D-dimer. So, lumen compressibility was assessed by pressure of the probe; RVT diameter was taken by measuring the distance between the anterior and posterior walls of the vein, on freeze frame of B-mode images, during compression with the ultrasound probe. RVT was arbitrarily scored as "absent" when the figure was $40 \%$ or less of the vein diameter.

All patients with present RVT at the beginning of this study were excluded D-dimer was assessed using the VIDAS® D_Dimer exclusion II TM (DEX2) qualitative, whole blood method assay (BIOMÉRIEX , Marcy, France; cut-off: $500 \mathrm{ng} / \mathrm{ml}$ ) by technicians unaware of the clinical characteristics of the patients. Anticoagulation was stopped on the same day and the next visit was scheduled after 30 days. Patients with recurrent events before the 30 day visit were excluded from further analysis. 
At the 30-day visit, venous blood samples were taken for D-dimer repeated test. All enrolled patients (with and without RVT) were stopped OAT.

\section{Study outcomes and follow-up}

Patients were followed for about 11 months after OAT discontinuation. Study outcomes were recurrent VTE. Patients were trained to contact the clinical center if symptoms developed suggestive of VTE or bleeding. In cases of recurrence, results of CUS were compared with those of the previous examination. Diagnosis of recurrent DVT was made if a previously fully compressible segment (contralateral or ipsilateral) became no longer compressible or if an increase of $40 \%$ or more in the diameter of the residual thrombus during compression was detected. In patients with suspected pulmonary embolism, diagnosis of VTE recurrence was based on results of D-dimer assay, pulmonary CT angiography or pulmonary ventilation/perfusion scanning. Although in case of low clinical pretest probability, D-dimer was measured and if normal, patients were not investigated further. In case of a suspected recurrence in the contralateral leg of the index event, patients with negative CUS and normal D-dimer (cut-off: $500 \mathrm{ng} / \mathrm{ml}$ ) were not investigated further. In case of negative CUS and abnormal D-dimer, a repeated CUS was scheduled after 5-7 days. If the repeated CUS was negative, patients were not investigated further. In case of a suspected DVT recurrence in the same leg of the index event, CUS results were compared with the results of the last available previous CUS. If the CUS was non-diagnostic and D-dimer normal, a repeated CUS was scheduled after 5-7 days.

We scheduled a follow-up visit at 1, 6 and 12 months after stopping of VKA for all patients. At each follow-up visit, we examined patients and inquired about health status, clinical symptoms or signs of recurrent VTE, bleeding, post-thrombotic manifestations, adherence to treatment, and concomitant analgesic or antiinflammatory therapy.

Patients received a card with the telephone numbers of the thrombosis centers and were instructed to return to the study center if clinical manifestations suggestive of recurrent venous thrombosis in either leg (edema, redness, tenderness, pain, or swelling), or pulmonary embolism (dyspnea, chest pain, or tachycardia) occurred. If this was the case, we invited patients to the Alzahra hospital (research center) for further diagnostic procedures. At first, all patients were requested to wear $40 \mathrm{mmHg}$ elastic stockings for more than two years. In case of a suspected recurrent event, D-dimer was sampled only for diagnostic purposes.

\section{Statistical analysis}

We used the chi-square test to compare the rate of persistent residual venous thrombosis at the last ultrasonography assessment and also abnormal D-dimer at 0, 1 months between the 2 groups. Differences between groups were assessed by the Fisher's exact test, chi-square test (Yates' correction) or $\mathrm{t}-$ test when appropriate. Approximate 95\% confidence intervals (95\% CI) were calculated based on the binomial distribution. Hazard ratio was calculated to assess the risk for recurrent VTE for patients with abnormal D-dimer versus those with normal D-dimer at both T0 and T1 versus those without. Kaplan-Meier estimates and 95\% CI were calculated to assess the risk for recurrent VTE for patients with abnormal D-dimer versus those with normal D-dimer at both $\mathrm{T} 0$ and $\mathrm{T} 1$.

A two-sided $\mathrm{p}$-value equal to or less than 0.05 was considered as statistically significant. The SPSS software package (13.0 release, Chicago, IL, USA) was used for data processing.

\section{Results}

\section{Patients}

A total of 68 eligible patients were enrolled since March 2011 at medical clinics of Alzahra hospital. Figure 1 shows the study flow chart of the 68 patients included in the study. After excluding four patients (two patients need to using long-term oral anticoagulation, and another two patients were excluded for lost to their first follow-up), 64 patients were enrolled at the end of first month. Ddimer and CUS at first was normal in 28 patients (44\%) while the remaining 36 patients had abnormal D-dimer but normal CUS while both groups were not consuming their anticoagulant. The baseline characteristics of the two groups of patients are reported in Table 1. Patients with normal D-dimer results were more likely to be younger

\section{Follow-up}

Total follow-up was 52.5 patient-years. Median follow-up was 11 months (95\% CI: 6-17 months). A minimum follow-up of 12 months was available in 44 patients $(68 \%)$. We have no reports of D-dimer at T1 in two patients but these patients were returned to our study in the next follow-up. In the extended follow-up, no patients were lost.

\section{Outcomes}

\section{Recurrent venous thromboembolism}

During follow-up, three recurrent events were recorded (4.6\% of patients - 95\% CI: 3-7\%) (Table 2). Recurrent events were ipsilateral in all cases. Among these patients, all had an abnormal D-dimer at either T0 and/or T1. The recurrence rate was higher in males than in females $(8.6 \%$ vs. $2.2 \%)$ with an abnormal Ddimer at T0 and/or T1 with a multivariate hazard ratio of 2.1

Table 1: The baseline characteristics of the two groups of patient

\begin{tabular}{|l|l|l|l|l|l|l|}
\hline & \multicolumn{3}{|c|}{ D-dimer at T1 } & \multicolumn{3}{c|}{ D-dimer at T0 } \\
\hline & Total & Abnormal & Normal & Total & Abnormal & Total \\
\hline N (\% of total) & 28 & 36 & 64 & 26 & 36 & $62 \mathrm{a}$ \\
\hline Male (\%) & $12(54)$ & $20(55)$ & $32(50)$ & $14(53)$ & $18(50)$ & $28(51)$ \\
\hline Age median Years (\% over 65 y) & $42(18)$ & $57(30)$ & $50(22)$ & $44(30)$ & $55(39)$ & $50(35)$ \\
\hline VKA duration mean months & $8.3 \pm .6$ & $7.7 \pm .4$ & $8.1 \pm .3$ & 8.7 & 8.4 & 8.5 \\
\hline Recurrence rate (\%) & $0(0)$ & $3(8.3)$ & $3(5.5)$ & $0(0)$ & $3(8.8)$ & $3(5.8)$ \\
\hline Follow-up Mean $\mathbf{\pm 9 5 \% C I ~ m o n t h s ~}$ & $10.8 \pm .7$ & $9.9 \pm .5$ & $10.2 \pm .4$ & $10.8 \pm .8$ & $9.8 \pm .5$ & $10.2 \pm .4$ \\
\hline
\end{tabular}


(95\% 1.2-7; $\mathrm{p}=0.04)$. But the rate of recurrence did not differ in relation to VKA duration among the patients who had been treated for $\leq 5$ or $>5$ months, respectively (hazard ratio 1.4: $95 \%$ CI: 0.8-4.8; $\mathrm{p}=0.06)$. Patients older than 65 years had a higher rate of events than younger patients (2/12: $16 \%$; 95\% CI: 4-24\% vs. $1 / 52$ : $2 \%$; $95 \%$ CI: $0.05-3 \%$ ) and hazard ratio was about 3.8 ; (95\% CI: $1.95-8.4 \mathrm{p}=0.04)$.

Table 2 shows the rate of primary outcomes by treatment assignment. Event rates were significantly higher in patients with an abnormal D-dimer did not resume anticoagulation drugs than those with a normal D-dimer.

\section{D-dimer at T0 and T1}

Patients with recurrences had higher mean D-dimer at both T0 and T1 when compared with those without recurrences but the difference was significant only for D-dimer at T1 (Mean \pm SE; $593 \pm 81$ vs. $343 \pm 35 \mathrm{ng} / \mathrm{ml}$ at $\mathrm{T} 0, \mathrm{p}=0.04 ; 638 \pm 57 \mathrm{ng} / \mathrm{ml}$ vs. $435 \pm$ $35 \mathrm{ng} / \mathrm{ml}$ at $\mathrm{T} 1, \mathrm{p}<0.01)$.

The characteristics of patients according to D-dimer at T0 and T1 classified as normal or abnormal (cut-off $500 \mathrm{ng} / \mathrm{ml}$ ) are shown in Table 1. All patients who had an abnormal D-dimer at T0 had also an abnormal D-dimer at T1. The rate of abnormal D-dimer increased significantly at T1 when compared to T0 $(\mathrm{p}<0.01)$. The characteristics of subjects with an abnormal or normal D- dimer either at T0 or T1 were similar except for a significant older age among subjects with abnormal D-dimer at both time points and a higher rate of males with a normal than with an abnormal Ddimer at $\mathrm{T} 1$.

The cumulative incidence of recurrence for $\mathrm{D}$-dimer both at T0 and T1 are shown in Figure 2A and B.

\section{Deaths}

During follow-up, two patients died (3\%). The cause of death was attributed to VTE in only one subject and to ischemic heart disease in another. All these patients had abnormal D-dimer at zero time (table 3)

\section{Discussion}

In this single centre study, we have evaluated a population of patients with idiopathic DVT presenting to a general hospital thrombosis center.

In our cohort of DVT patients, the annual risk of recurrence with an abnormal D-dimer, either during or at one month after VKA withdrawal, was $4.6 \%$ which is much lower to the annual risk of recurrence in most studies with idiopathic and provoked VTE.

Table 2: Rate of primary outcomes by treatment assignment according to D-dimer at zero time

\begin{tabular}{|l|l|l|l|}
\hline Outcomes & Normal-Dd0 & Abnormal-Dd0 & Total \\
\hline Death $-\mathrm{n} / \mathrm{n}$ total $(\%)$ & $0 / 28(0)$ & $2 / 36(5.5)$ & $2 / 64(3.1)$ \\
VTE related & & $1 / 36(\sim 2.7)$ & \\
Ischemic heart disease related & & $1 / 36(\sim 2.7)$ & \\
Recurrence (all DVT) & $0 / 28$ & $3 / 36) 8.3)$ & $3 / 64(4.6)$ \\
\hline
\end{tabular}

Fig. 2: hazard ratio for D-Dimer at T0 and T1 for DVT patients

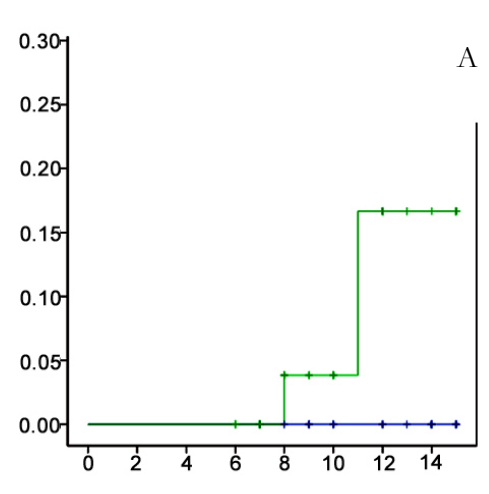

hazard ratio for D-Dimer at T0

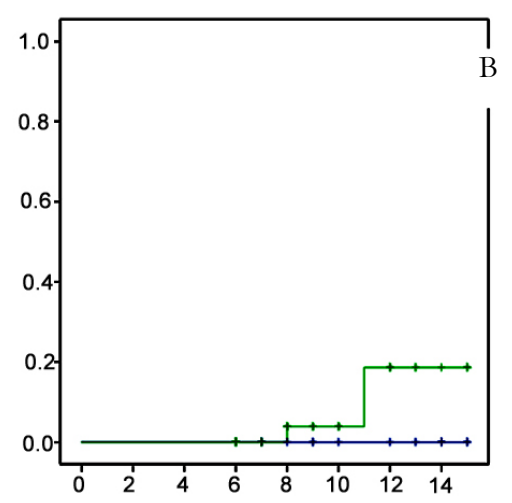

hazard ratio for D-Dimer at T1

Table 3: Calculating sensitivity and specificity and likelihood ratio for quantitative D-Dimer test with cut point of about $500 \mathrm{ng} / \mathrm{ml}$ in predicting DVT recurrence

\begin{tabular}{|l|l|l|l|l|l|l|}
\hline & Sensitivity & Specificity & $\begin{array}{c}\text { Positive predictive } \\
\text { value }\end{array}$ & $\begin{array}{c}\text { Negative predictive } \\
\text { value }\end{array}$ & $\begin{array}{c}\text { Positive Likelihood } \\
\text { ratio }\end{array}$ & $\begin{array}{c}\text { Negative likelihood } \\
\text { ratio }\end{array}$ \\
\hline D-Dimer at T0 & $100 \%$ & $67.3 \%$ & $14.3 \%$ & $100 \%$ & 1.51 & 1.41 \\
\hline D-Dimer at T1 & $100 \%$ & $79 \%$ & $23 \%$ & $100 \%$ & 1.28 & 1.25 \\
\hline
\end{tabular}


[5]. Paolo Prandoni [7] reported that the cumulative incidence of recurrent VTE was $11 \%$ and Young [2] in 2006 showed that recurrence rate was only 4\%(2-7. According to patient selection, our results were contributed to idiopathic DVT results as shown in Young study.

Our results also demonstrated that in subjects with idiopathic DVT, an abnormal D-dimer, either at the end of treatment or at one month after VKA withdrawal, is associated with a higher risk of recurrence than a normal D-dimer.

In 2010, PROLOG study, confirmed that an abnormal D-dimer measured one month after VKA withdrawal is associated with a significantly higher risk of recurrence than normal D-diner in patients with proximal DVT of the lower limbs [9]. Besides, Bruinstroop et all, in 2009, showed that elevated D-dimer level measured 1 month after discontinuation of OAT identify patients with idiopathic VTE at a significantly higher risk of recurrent VTE $[10]$.

Results of the extended follow-up of the PROLNG study confirmed the benefit of prolonging VKAS in patients with an abnormal D-dimmer at 1 month after anticoagulation withdrawal. These results strengthen the finding that D-dimmer is associated with the individual risk of recurrence after a first episode of unprovoked VTE. The optimal course of therapy in patients with normal D-dimmer remains unclear also with the extended followup.

Contrary, Baglin et al, evaluated patients with non-surgical provoked VTE over a median follow up of 36 month. Results showed that post anticoagulation D-dimmer was not predictive of recurrent evens over the follow up [11].

The negative predictive value of D-dimmer (NPV) in our study was $100 \%$. Which was near to results of verhovesk at all, (NPV of D-dimmer was 92\%) and in the study by Bruinstroop and Palareti were $93 \%[10,12,13]$.

The AESPUS and DACUS studies was conducted in patients with both provoked and unprovoked DVT, but only patients with unprovoked DVT were enrolled in our study $[3,14]$. Neither the AESPUS nor the DACUS studies evaluated RVO in combination with D dimmer, but PROLONG study do it. PROLONG study is the only randomized study evaluating both parameters $[9,15]$.

In our study no effect of the presence of RVO was observed possibly due to the small number of events and the small sample size. The study also demonstrates that patients with a confirmed diagnosis of DVT have significant cumulative mortality with rates of $3 \%$ at the end of first year which was similar to Young results, with $4 \%, 12 \%$ and $27 \%$ at 1,2 , and 5 years, respectively [2].

In conclusion, this study confirms that D-dimmer at the time of withdrawal and 1 month after VKA discontinuation is an independent risk factor for recurrent VTE after a single episode of idiopathic proximal DVT of the lower limbs and very useful for the clinicians in selecting patients at high risk of recurrent VTE.

\section{Study Limitations}

This study has a number of important limitations. The sample size was not calculated to assess the relative risk of cardiovascular events, death and cancer.. Future studies should focus on determining optimal D-dimer cut-off level and optimize multi risk factor model and duration of extended therapy in patients with elevated D-dimmer levels.

\section{References}

[1]. Anderson FA Jr, Wheeler HB, Goldberg RJ, Hosmer DW, Patwardhan NA, et al (1991) population-based perspective of the hospital incidence and case fatality rates of deep vein thrombosis and pulmonary embolism: Arch Intern Med;151:933-8.

[2]. Young L, Ockelford P, Milne D, Rolfe-Vyson V, Mckelvie S, et al (2006) Post-treatment residual thrombus increases the risk of recurrent deep vein thrombosis and mortality. J Thromb Haemost; 4: 1919- 24.

[3]. Siragusa S, Malato A, Anastasio R, Cigna V, Milio G (2008) Residual vein thrombosis to establish duration of anticoagulation after a first episode of deep vein thrombosis: the Duration of Anticoagulation based on Compression UltraSonography (DACUS) study. Blood; 112: 511-15.

[4]. Carrier M, Rodger M .A, Wells P.S, Righini M, Le Gal G (2011) Residual vein obstruction to predict the risk of recurrent venous thromboembolism in patients with deep vein thrombosis: a systematic review and meta-analysis. J Thromb Haemost; 9: 1119-25

[5]. Cosmi B, Legnani C, Cini M,Guazzaloca G, Palareti G (2011) D-dimer and residual vein obstruction as risk factors for recurrence during and after anticoagulation withdrawal in patients with a first episode of provoked deepvein thrombosis. Thromb Haemost; 105: 837-845

[6]. Cosmi B, Legnani C, Iorio A, Pengo V, Ghirarduzzi A (2010) Residual venous obstruction, alone and in combination with $\mathrm{D}$-dimer, as a risk factor for recurrence after anticoagulation withdrawal following a first idiopathic deep vein thrombosis in the prolong study. Eur J Vasc Endovasc Surg; 39: 356-65.

[7]. Prandoni P, Noventa F, Ghirarduzzi A. (2007) The risk of recurrent venous thromboembolism after discontinuing anticoagulation in patients with acute proximal deep vein thrombosis or pulmonary embolism. A prospective cohort study in 1,626 patients. Haematologica; 92: 199-205.

[8]. Poli D, Antonucci E, Ciuti G, Abbate R, Prisco D (2008) Combination of D-dimer, F1p2 and residual vein obstruction as predictors of VTE recurrence in patients with first VTE episode after OAT withdrawal. J Thromb Haemost;6:708-10.

[9]. Cosmi B, Legnani C, Tosetto A (2010) PROLONG Investigators (on behalf of Italian Federation of Anticoagulation Clinics). usefulness of repeated D-dimer testing after stopping anticoagulation for a first episode of unprovoked venous thromboembolism:the PROLONG II prospective study. Blood; 115: 481-488.

[10]. Bruinstroop E, Klok FA, Van De Ree MA, Oosterwijk FL, Huisman MV (2009) Elevated D-dimer levels predict recurrence in patients with idiopathic venous thromboembolism: a meta-analysis. J Thromb Haemost;7: 611-618.

[11]. Baglin T, Luddington R, Brown K (2003) Incidence of recurrent venous thromboembolism in relation to clinical and thrombophilic risk factors: prospective cohort study. Lancet; 362: 523-526

[12]. Verhovsek M, Douketis JD, Yi Q, Shrivastava S, Tait RC, Baglin T (2008) Systematic review: D-dimer to predict recurrent disease after stopping anticoagulant therapy for unprovoked venous thromboembolism. Ann Intern Med; 149: 481-90.

[13]. Palareti G, Legnani C, Cosmi B (2002) Risk of venous thromboembolism recurrence: high negative predictive value of D-Dimer performed after oral anticoagulation is stopped. Thromb Haemost 2002; 87: 7-1219.

[14]. Prandoni P, Prins MH, Lensing AW, Ghirarduzzi A, Ageno W. et al (2009) AESOPUS Investigators. Residual thrombosis on ultrasonography to guide the duration of anticoagulation in patients with deep venous thrombosis: a randomised trial. Ann Intern Med May 5;150(9):577-85

[15]. Cosmi B, Legnani C, Tosetto A (2010) Comorbidities, alone and in combination with $\mathrm{D}$-dimer, as risk factors for recurrence after a first episode of unprovoked venous thromboembolism in the extended follow-up of the PROLONG study. Thromb Haemost;103: 1152-1160. 